\title{
Review of: "Whole-brain in vivo base editing reverses autistic-like behaviors in mice"
}

\author{
Jong Hoon Ryu ${ }^{1}$
}

1 Kyung Hee University

Potential competing interests: The author(s) declared that no potential competing interests exist.

Title: Whole-brain in vivo base editing reverses autistic-like behaviors in mice

In this manuscript, the authors investigated the effects of CRISPR-mediated cytidine base editor (CBE) system in autism spectrum disorder (ASD) mouse model associated with mutation in the MEF2C gene. The authors identified reduced the levels of MEF2C protein in MEF2C-L35P heterozygous mice and confirmed autism-like behaviors. The authors then showed that CBE reversed the amount of MEF2C protein and improved autism-like behavior. It is meaningful work, however, there are some points to revised before publication for this journal.

\section{Major point}

1. In this manuscript, the authors reported that MEF2C-L35P heterozygous mice spend a lot of time in the open arm in the elevated plus maze (EPM). The authors said that the reason MEF2C-L35P heterozygous mice spend more time in the open arm is because hyperactivity appeared rather than anxiety-like behavior. The authors showed that hyperactivity was improved in the open field test (OFT), but they did not explain the changes in the EPM after CBE treatment. To support their claims, the authors should show whether the time spend on the open arm in the EPM was reduced or not.

2. In the method session, the authors explained that mild light intensity was adjusted to 50 lux applied in marble burying, but data on marble burying is not included in this manuscript. Therefore, the authors should present data about marble burying test.

3. In this manuscript, the authors investigated only the behavior of MEF2C-L35P heterozygous mice before and after CBE treatment and the expression level of MEF2C protein. However, other indicators related to ASD have not been confirmed. It has been acknowledged that abnormal activation of microglia and astrocyte appears in ASD patients (Suzuki K, et al., JAMA Psychiatry. 2013 Jan;70(1):49-58.; Vargas DL, et al., Ann Neurol. 2005 Jan;57(1):67-81.), and dysfunction of microglia and astrocyte induces ASD-like behaviors in mice (Xu ZX, et al. Nat Commun. 2020 Apr 14;11(1):1797.; Wang Q, et al. Nat Commun. 2021 May 31;12(1):3321.). Therefore, the authors should identify their biomarkers Iba1 and GFAP. In addition, the authors should identify inflammatory cytokines such as IL-1 $\beta$ and IL- 6 because the secretion of inflammatory cytokines is increased in ASD patients (Masi A, et al., Mol Psychiatry. 2015 
Apr;20(4):440-6.). 